\title{
MicroRNA-29c-3p acts as a tumor suppressor gene and inhibits tumor progression in hepatocellular carcinoma by targeting TRIM31
}

\author{
TAO LV, LI JIANG, LINGXIANG KONG and JIAYIN YANG \\ Department of Hepato-Biliary-Pancreatic Surgery, West China Hospital of Sichuan University, \\ Chengdu, Sichuan 610041, P.R. China
}

Received July 19, 2019; Accepted December 10, 2019

DOI: $10.3892 /$ or.2020.7469

\begin{abstract}
Aberrant expression of microRNAs (miRNAs) has been widely reported in many malignant tumors, and dysregulated miRNAs play an important role in the malignant progression of tumors. It has been reported that miR-29c-3p expression is dysregulated in tumors and promotes the development of tumors, especially in hepatocellular carcinoma (HCC). However, the specific mechanism of miR-29c-3p in HCC is not clear. The present study demonstrated that miR-29c-3p was expressed at low levels in HCC patients and cell lines and that its decreased expression was closely related to poor prognosis of HCC patients. Overexpression of miR-29c-3p could significantly inhibit the proliferation and migration of HCC cells in vitro and suppress the HCC tumor growth in vivo. The luciferase reporter assay demonstrated that miR-29c-3p directly bound to tripartite motif containing 31 (TRIM31) and suppressed TRIM31 expression. Finally, upregulation of TRIM31 could partially abolish the tumor suppressing roles of miR-29c-3p in HCC. Overall, miR-29c-3p, as a tumor suppressor gene, was revealed to inhibit the malignant progression of HCC by reducing the expression of TRIM31 and may be used as a potential therapeutic target for the precise treatment of HCC.
\end{abstract}

\section{Introduction}

As the second most frequent cancer in the world, hepatocellular carcinoma (HCC) causes 788,000 deaths each year (1). In addition, HCC is also the main cause of tumor death in China. At the time of diagnosis, most HCC patients are at more advanced stages, which are accompanied by intrahepatic and extrahepatic metastases, and the prognosis is poor. At present, there is a lack

Correspondence to: Professor Jiayin Yang, Department of Hepato-Biliary-Pancreatic Surgery, West China Hospital of Sichuan University, 37 Guoxue Road, Chengdu, Sichuan 610041, P.R. China E-mail: jiayinyang5@163.com

Key words: microRNA-29c-3p, TRIM31, hepatocellular carcinoma, proliferation, migration of effective prevention and treatment measures clinically. The 5 -year metastasis and recurrence rate after radical resection is as high as $61.5 \%$ (2). Recurrence and metastasis of tumors are still the main causes of treatment failure in HCC, causing a heavy burden on families and society (3). Therefore, it is urgent to elucidate the mechanism of recurrence and metastasis, and provide new treatment strategies for $\mathrm{HCC}$.

MicroRNAs (miRNAs) are single-stranded noncoding small RNA with a length of 21-23 nt (4). When miRNAs are loaded into the RNA-induced silencing complex (RISC), they can play a role in regulating gene expression. Through their seed sequence (5-terminal 2-8 nucleotides), miRNAs can identify binding sites on target gene mRNA 3'-UTRs and carry recruit RISC to play its role, which results in transcriptional inhibition, cleavage and degradation of Mrna (5). It has been reported that miRNA dysfunction is closely related to the invasion and metastasis of tumors (6,7). In HCC, miR-424-5p was revealed to inhibit TRIM29 expression, and then regulate HCC cell proliferation and invasion by inhibiting gene expression of proliferation- and apoptosis-related indicators (8). A recent study revealed that miR-505 inhibited the malignant development of non-small cell lung cancer via the MAP3K3-mediated AKT-NFאB signaling pathway (9). In gastric cancer, miR-125a inhibited the invasion and migration of tumor cells by regulating the expression of HAS1 by targeting STAT3 (10). Recent studies have revealed that miR-29c-3p is abnormally expressed in many malignant tumors including gastric cancer (11), colon cancer (12) and pancreatic cancer (13). A previous study revealed that miR-29c-3p was expressed at low levels in laryngeal squamous cell carcinoma (14). It was reported that miR-29c-3p overexpression led to decreased migration of GC cells in vitro and in vivo by suppressing the expression of KIAA1199 and several key proteins in the Wnt/ $\beta$-catenin and EGFR signaling pathways (15). miR-29c-3p regulates CRC cell proliferation and migration by regulating SPARC expression (16). A study also revealed that miR-29c-3p promoted the malignant development of HCC by regulating the methylation of LATS1 caused by DNMT3B and inhibiting the anticancer function of the Hippo signaling pathway (17). However, a single miRNA can regulate the expression of hundreds of target gene mRNAs after transcription. Therefore, the specific roles and molecular mechanisms of miR-29c-3p in HCC have not been fully elucidated $(18,19)$. 
In the present study, the expression of miR-29c-3p in HCC was revealed to be significantly decreased, and its low expression was closely related to the poor prognosis of HCC patients. Overexpression of miR-29c-3p could significantly inhibit the proliferation and migration of HCC cells. It was also confirmed that miR-29c-3p could inhibit the malignant progression of HCC by directly acting on tripartite motif containing 31 (TRIM31) to regulate tumor proliferation and migration-related factors.

\section{Materials and methods}

HCC patients and specimens. A total of $60 \mathrm{HCC}$ tissue samples were collected in this study, including tumor tissues and paired normal adjacent tissues, which were collected from January 2006 to July 2011 at the West China Hospital of Sichuan University and sample collection used liquid nitrogen for preservation. The histological diagnosis of all HCC samples was performed independently by two pathologists. All patients signed an informed consent form. The present study was approved by the Ethics Committee of West China Hospital, Sichuan University.

Cell culture and transfection. The liver cancer cells (HepG2 and MHCC-97H), which were assessed by STR profiling, used in the present study were obtained from the Institute of Biochemistry and Cell Biology (Chinese Academy of Sciences). All cell lines were cultured with high-glucose DMEM containing 10\% FBS (Hyclone; GE Healthcare Life Sciences) and $1 \%$ penicillin/streptomycin.

miR-29c-3p mimic (miR-29c-3p), miR-29c-3p inhibitor and miR-29c-3p control were obtained from Guangzhou RiboBio Co., Ltd. The TRIM31 overexpression vector pcDNA-TRIM31 and empty control vector pcDNA were constructed by Shanghai GenePharma Co., Ltd. Lipofectamine 3000 reagent (Invitrogen; Thermo Fisher Scientific, Inc.) was used for liver cancer cell (HepG2 and MHCC-97H) transfection according to the manufacturer's instructions.

Quantitative real-time polymerase chain reaction ( $q R T-P C R)$. Total RNA was extracted from HCC tissue samples and cell lines using TRIzol reagent (Takara Bio, Inc.) according to the manufacturer's protocol. miR-29c-3p expression was determined by a TaqMan MicroRNA Assay kit (Applied Biosystems; Thermo Fisher Scientific, Inc.). Total RNA was reverse-transcribed into cDNA using PrimeScript RT Reagent (Takara Bio, Inc.). qRT-PCR was performed using SYBR Premix Ex Taq II (Takara Bio, Inc.). The temperature protocol for qRT-PCR was as follows: $35^{\circ} \mathrm{C}$ for $5 \mathrm{~min}$, followed by $45^{\circ} \mathrm{C}$ for $40 \mathrm{~min}$ and $70^{\circ} \mathrm{C}$ for $5 \mathrm{~min}$. U6 and GAPDH were used as internal references. The sequences of the primers used for each gene are presented in Table SI. The mRNA expression of miR-29c-3p and TRIM31 was determined using the $2^{-\Delta \Delta \mathrm{Cq}}$ method (20).

Western blotting. Total protein was extracted using RIPA lysis buffer (Beyotime Institute of Biotechnology). Protein was quantified using the Bradford protein assay (Bio-Rad Laboratories, Inc.) with a NanoDrop spectrophotometer. A total of $25 \mu \mathrm{g} / \mathrm{well}$ of protein was electrophoresed on $10 \%$ sodium dodecyl sulfate polyacrylamide gel electrophoresis (SDS-PAGE). After the transfer was completed, the PVDF membranes were blocked with
$5 \%$ non-fat powdered milk at $37^{\circ} \mathrm{C}$ for $1 \mathrm{~h}$. Next, the membranes were incubated with anti-TRIM31 (1:2,000; ab98207; Abcam) and anti- $\beta$-actin antibody (1:5,000; ab179467; Abcam) at $4^{\circ} \mathrm{C}$ overnight. Subsequently, the PVDF membranes were incubated with horseradish peroxidase-conjugated secondary antibody $(1: 5,000 ;$ ab6721; Abcam) at room temperature for $1 \mathrm{~h}$. $\beta$-Actin was used as an internal reference. Finally, enhanced chemiluminescence (ECL) (Thermo Fisher Scientific, Inc.) was used to detect the expression of the target proteins. Quantity One software v4.6.5 (Bio-Rad Laboratories, Inc.) was used for densitometry, and the values are expressed relative to $\beta$-actin.

Cell Counting Kit-8 (CCK-8) assay. The transfected cells were inoculated into 96-well plates. After adding $10 \mu \mathrm{l} /$ well of CCK-8 solution (Dojindo Molecular Technologies, Inc.), the absorption was determined at $450 \mathrm{~nm}$ by microplate spectrophotometer. The OD values at $450 \mathrm{~nm}$ were detected at $0,6,12,24,48$ and $72 \mathrm{~h}$ according to the manufacturer's instructions.

5-Ethynyl-2'-deoxyuridine (EdU) assay. In brief, $5 \times 10^{3}$ cells/wells were plated in 96-well plates and cultured for $24 \mathrm{~h}$. Next, $4 \%$ ice formaldehyde was added for cell fixation for $30 \mathrm{~min}$ at room temperature, and the cells were permeabilized with $0.5 \%$ Triton X-100 solution for $20 \mathrm{~min}$. Cells were incubated with $\mathrm{EdU}(50 \mu \mathrm{M})$ for $2 \mathrm{~h}$ at $37^{\circ} \mathrm{C}$. After washing the cells three times with PBS, $1 \mathrm{X}$ ApolloR reaction cocktail $(100 \mu \mathrm{l})$ was added, and the reaction proceeded for $30 \mathrm{~min}$ at $37^{\circ} \mathrm{C}$ in the dark. Subsequently, $1 \mathrm{X}$ Hoechst 33342 (100 $\left.\mu \mathrm{l}\right)$ was used to stain the cell nuclei for $30 \mathrm{~min}$ at room temperature. Cell proliferation was analyzed using the mean number of the cells in each sample using a fluorescence microscope (Lionheart; BioTek Instruments, Inc.; magnification, x100).

Wound healing assay. The cells ( $1 \times 10^{5}$ cells/wells) were seeded into 6 empty plates, and after the cells were confluent (80-90\%), 3 lines were scratched with a $1 \mathrm{ml}$ pipette tip on the plate wells. Non-adherent cells were washed off with PBS. After 0, 24 and $48 \mathrm{~h}$, the distance between the wound edges was measured. Cells were examined under a Leica light microscope (Olympus Corp.; magnification, x40).

Cell migration assay. After $48 \mathrm{~h}$ of cell transfection, the cells were collected in a cell suspension and added to the upper chamber (Corning, Inc.) with $200 \mu \mathrm{l}$ of serum-free medium. Next, $500 \mu \mathrm{l}$ of medium containing $10 \%$ FBS was added to the lower chamber. After $24 \mathrm{~h}$ of culture, the non-migrating cells were wiped off with a cotton swab. The cells were fixed with $4 \%$ formaldehyde and then stained with $0.1 \%$ crystal violet, both at room temperature. The cells were washed three times with PBS and counted under a microscope.

Luciferase reporter assay. miRanda (http://www.microrna. org/microrna/home.do) and TargetScan (http://www.targetscan. org) were used to identify downstream target genes of miR-29c-3p. The wild-type TRIM31-3'UTR (WT) and mutant TRIM31-3'UTR (MUT) containing the putative binding site of miR-29c-3p were amplified by Shanghai GenePharma Co., Ltd., and cloned into the firefly luciferase-expressing pMIR-REPORT vector (OBiO Technology Corp., Ltd.). The luciferase reporter vector and miR-29c-3p mimic or miR-NC were transiently 

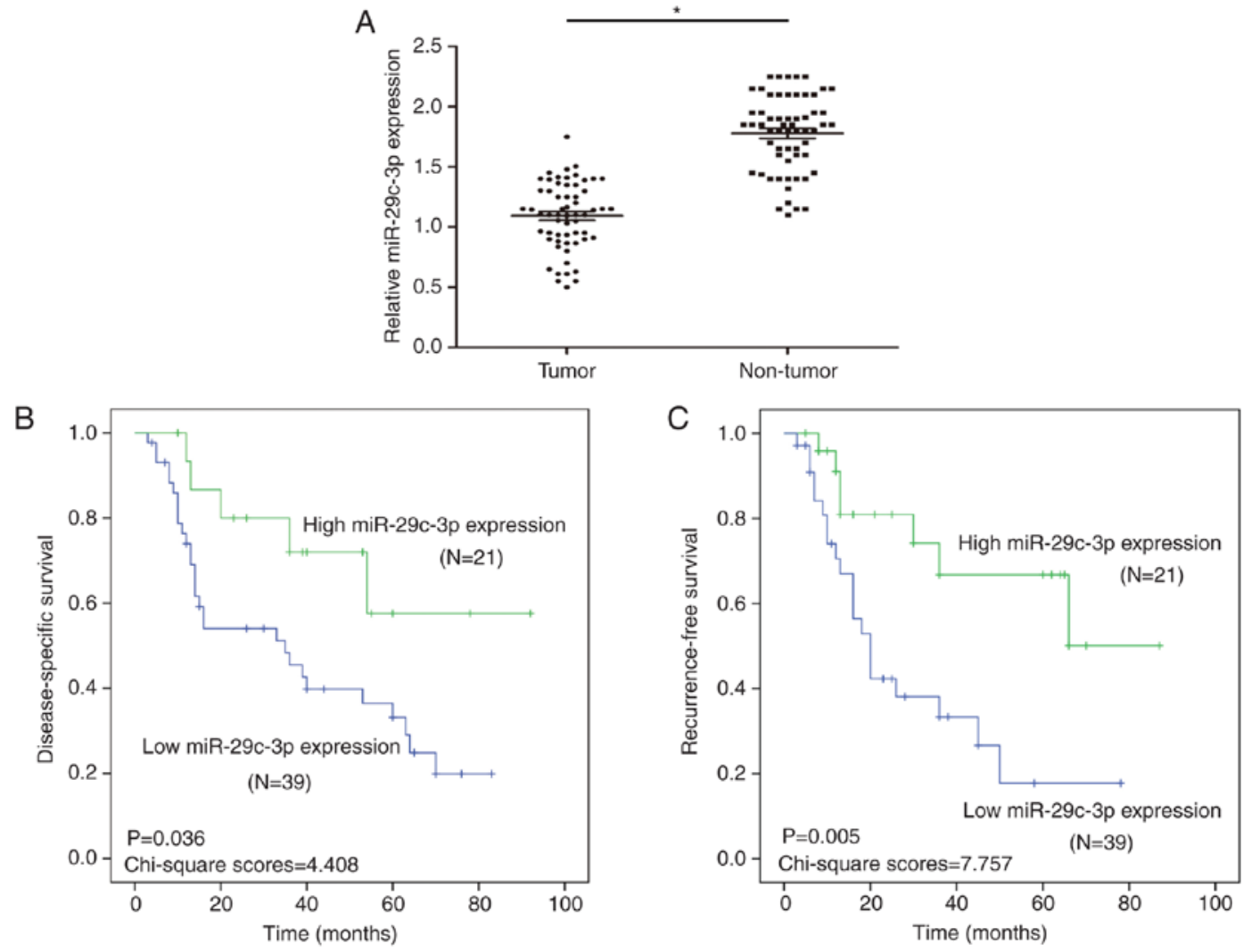

Figure 1. miR-29c-3p is downregulated in HCC. (A) The results of qRT-PCR revealed the expression level of miR-29c-3p in HCC and adjacent non-cancerous tissues $(n=60)$. (B) Kaplan-Meier analysis of DSS between HCC patients with high and low miR-29c-3p expression. (C) Kaplan-Meier analysis of RFS between HCC patients with high and low miR-29c-3p expression. " $\mathrm{P}<0.05$ compared to the control. HCC, hepatocellular carcinoma; DSS, disease-specific survival; RFS, recurrence-free survival.

co-transfected using Lipofectamine 3000. After transfection for $24 \mathrm{~h}$, luciferase assays were performed using the Luciferase Reporter Assay System (GloMax) according to the manufacturer's protocol.

Mouse xenograft tumor model. BALB/c-nu mice (age, 5 weeks; sex, male; weight, 20-22 g) were purchased from Shanghai Experimental Animal Center and housed in a sterile room at the Experimental Animal Center of West China Hospital of Sichuan University at $25^{\circ} \mathrm{C}$ and $40-70 \%$ humidity, with a 12 -h light/dark cycle and free access to food and water. All animal experiments were performed in accordance with the institutional guidelines, and the method of euthanasia was cervical dislocation (when the heart stopped completely, the mouse was determined as dead). Body weight loss $>20 \%$ was assumed to be a humane endpoint for euthanasia. After transfecting the miR-29c-3p mimic into liver cancer cells (HepG2 and MHCC-97H) and culturing for $48 \mathrm{~h}$, the transfected liver cancer cells (HepG2 and MHCC-97H) $\left(5 \times 10^{6}\right)$ were subcutaneously injected into the left hip flanks of the mice. The tumor volume was calculated according to the following formula: Volume $=\left(\right.$ length $\mathrm{x}$ width $\left.^{2}\right) / 2$. Tumor sizes (the length and width of tumor nodules) were measured every 5 days. All animal experiments were approved by the Animal Care Ethics Review Committee of West China Hospital of Sichuan University. Then, 50 days after injection, the mice were sacrificed, and tumors were collected for analysis. The tumor experiments ended when tumor diameters were $<20 \mathrm{~mm}$ (the maximum tumor volume was $397 \mathrm{~mm}^{3}$ ).
Statistical analysis. All data are presented as the means \pm standard deviation (SD). Statistical analysis of data was performed using GraphPad Prism version 6.0 (GraphPad Software, Inc.) or SPSS 20.0 software (IBM Corp.). Statistical differences were analyzed by Student's t-test, while the significance of differences between multiple groups was determined by one-way analysis of variance (ANOVA), followed by the Newman-Keuls test, and repeated measures ANOVA. The Kaplan-Meier method was used to assess disease-specific survival (DSS) and recurrence-free survival (RFS), the log-rank test and chi-squared analysis were used to analyze the differences between the curves. Univariate and multivariate Cox regression analyses were carried out to determine the prognostic significance of miR-29c-3p and TRIM31 expression. The correlation between miR-29c-3p and TRIM31 expression was evaluated by Spearman's correlation analysis. $\mathrm{P}<0.05$ was considered to indicate a statistically significant difference.

\section{Results}

Expression of miR-29c-3p is reduced in HCC tissues and the low expression of miR-29c-3p is closely related to poor prognosis in HCC. To study the expression of miR-29c-3p in HCC, qRT-PCR analysis of $60 \mathrm{HCC}$ and paired normal samples was performed. The results revealed that the expression of miR-29c-3p was downregulated in the HCC tissues compared with the paired normal samples (Fig. 1A). 
Table I. Correlations between miR-29c-3p and clinicopathological features of HCC patients $(n=60)$.

\begin{tabular}{|c|c|c|c|c|}
\hline \multirow[b]{2}{*}{ Variables } & \multirow[b]{2}{*}{ No. of cases } & \multicolumn{2}{|c|}{ miR-29c-3p expression } & \multirow[b]{2}{*}{ P-value } \\
\hline & & Low $(\mathrm{n}=39)$ & $\operatorname{High}(\mathrm{n}=21)$ & \\
\hline Age (years) & & & & 0.515 \\
\hline$<50$ & 28 & 17 & 11 & \\
\hline$\geq 50$ & 32 & 22 & 10 & \\
\hline Sex & & & & 0.548 \\
\hline Male & 34 & 21 & 13 & \\
\hline Female & 26 & 18 & 8 & \\
\hline Tumor size (cm) & & & & 0.013 \\
\hline$\leq 5$ & 27 & 13 & 14 & \\
\hline$>5$ & 33 & 26 & 7 & \\
\hline AFP (ng/ml) & & & & 0.399 \\
\hline$\leq 20$ & 27 & 16 & 11 & \\
\hline$>20$ & 33 & 23 & 10 & \\
\hline TNM stage & & & & 0.003 \\
\hline $\mathrm{I} / \mathrm{II}$ & 27 & 12 & 15 & \\
\hline III/IV & 33 & 27 & 6 & \\
\hline Liver cirrhosis & & & & 0.664 \\
\hline Presence & 32 & 20 & 12 & \\
\hline Absence & 28 & 19 & 9 & \\
\hline HBsAg & & & & 0.254 \\
\hline Positive & 37 & 22 & 15 & \\
\hline Negative & 23 & 17 & 6 & \\
\hline Vascular invasion & & & & 0.183 \\
\hline Presence & 27 & 20 & 7 & \\
\hline Absence & 33 & 19 & 14 & \\
\hline Multiplicity & & & & 0.047 \\
\hline Single & 24 & 12 & 12 & \\
\hline Multiple ( $\geq 2$ ) & 36 & 27 & 9 & \\
\hline Intrahepatic metastasis & & & & 0.217 \\
\hline Presence & 25 & 14 & 11 & \\
\hline Absence & 35 & 25 & 10 & \\
\hline
\end{tabular}

HCC, hepatocellular carcinoma.

Through the analysis of clinical prognosis in $\mathrm{HCC}$, it was revealed that HCC patients with low miR-29c-3p expression had significantly shorter DSS than those with high miR-29c-3p expression (Fig. 1B). Moreover, HCC patients with low miR-29c-3p expression had significantly shorter RFS than those with high miR-29c-3p expression (Fig. 1C).

In addition, miR-29c-3p expression was positively correlated with tumor size $(\mathrm{P}=0.013)$, TNM stage $(\mathrm{P}=0.003)$ and multiplicity $(\mathrm{P}=0.047)$ (Table I). By univariate analysis, TNM stage $(\mathrm{P}=0.014)$, tumor size $(\mathrm{P}=0.009)$, multiplicity $(\mathrm{P}=0.046)$ and miR-29c-3p $(\mathrm{P}=0.012)$ were significantly associated with DSS, and TNM stage $(\mathrm{P}=0.010)$, tumor size $(\mathrm{P}=0.005)$, multiplicity $(\mathrm{P}=0.034)$ and miR-29c-3p $(\mathrm{P}=0.019)$ were significantly associated with RFS (Tables II and III). The multivariate model revealed that DSS was significantly dependent on TNM stage
$(\mathrm{P}=0.020)$, tumor size $(\mathrm{P}=0.016)$ multiplicity $(\mathrm{P}=0.035)$ and miR-29c-3p $(\mathrm{P}=0.017)$, while TNM stage $(\mathrm{P}=0.015)$, tumor size $(\mathrm{P}=0.013)$, multiplicity $(\mathrm{P}=0.027)$ and miR-29c-3p $(\mathrm{P}=0.014)$ were significantly associated with RFS (Tables II and III), which indicated that miR-29c-3p was an independent prognostic factor for DSS and RFS in patients with HCC.

Upregulated expression of miR-29c-3p suppresses cell proliferation and migration in HCC cells. Gain-of-function experiments were used for the analysis of miR-29c-3p expression in liver cancer cells (MHCC-97H and HepG2). qRT-PCR results confirmed that the expression of miR-29c-3p was significantly increased after transfection of the miR-29c-3p mimic in liver cancer cells (MHCC-97H and HepG2) (Fig. 2A). The CCK-8 results revealed that overexpression of miR-29c-3p 
Table II. Univariate and multivariate analysis of various prognostic variables influencing DSS in HCC patients.

\begin{tabular}{|c|c|c|c|c|c|}
\hline \multirow[b]{2}{*}{ Variables } & \multirow[b]{2}{*}{$\mathrm{n}$} & \multicolumn{2}{|c|}{ Univariate analysis } & \multicolumn{2}{|c|}{ Multivariate analysis } \\
\hline & & $\mathrm{HR}(95 \% \mathrm{CI})$ & P-value & $\operatorname{HR}(95 \% \mathrm{CI})$ & P-value \\
\hline Sex & & $0.958(1.306-3.485)$ & 0.754 & & \\
\hline Male & 34 & & & & \\
\hline Female & 26 & & & & \\
\hline Age (years) & & $0.648(0.594-1.751)$ & 0.392 & & \\
\hline$<50$ & 28 & & & & \\
\hline$\geq 50$ & 32 & & & & \\
\hline Tumor size (cm) & & $0.598(0.678-1.985)$ & 0.009 & $0.757(1.032-2.485)$ & 0.016 \\
\hline$\leq 5$ & 27 & & & & \\
\hline$>5$ & 33 & & & & \\
\hline $\operatorname{AFP}(\mathrm{ng} / \mathrm{ml})$ & & $1.864(1.358-2.603)$ & 0.561 & & \\
\hline$\leq 20$ & 27 & & & & \\
\hline$>20$ & 33 & & & & \\
\hline HBsAg & & $0.894(0.754-2.625)$ & 0.435 & & \\
\hline Positive & 37 & & & & \\
\hline Negative & 23 & & & & \\
\hline TNM stage & & $1.385(0.677-1.807)$ & 0.014 & $1.048(1.986-3.842)$ & 0.020 \\
\hline $\mathrm{I} / \mathrm{II}$ & 27 & & & & \\
\hline III/IV & 33 & & & & \\
\hline Multiplicity & & $0.481(0.539-1.750)$ & 0.046 & $0.780(0.954-1.874)$ & 0.035 \\
\hline Single & 24 & & & & \\
\hline Multiple ( $\geq 2)$ & 36 & & & & \\
\hline Vascular invasion & & $1.235(0.953-4.473)$ & 0.842 & & \\
\hline Presence & 27 & & & & \\
\hline Absence & 33 & & & & \\
\hline Liver cirrhosis & & $0.684(0.465-1.383)$ & 0.796 & & \\
\hline Presence & 32 & & & & \\
\hline Absence & 28 & & & & \\
\hline Intrahepatic metastasis & & $0.597(1.346-3.846)$ & 0.480 & & \\
\hline Presence & 25 & & & & \\
\hline Absence & 35 & & & & \\
\hline miR-29c-3p expression & & $1.459(0.734-1.975)$ & 0.012 & $1.103(1.657-3.189)$ & 0.017 \\
\hline Low & 39 & & & & \\
\hline High & 21 & & & & \\
\hline
\end{tabular}

DSS, disease-specific survival; HCC, hepatocellular carcinoma.

inhibited the proliferation of liver cancer cells (MHCC-97H and HepG2) (Fig. 2B). As revealed in Fig. 2C, EdU assays indicated that overexpression of miR-29c-3p significantly inhibited the uptake of EdU in liver cancer cells (MHCC-97H and HepG2). Wound healing assays revealed that overexpression of miR-29c-3p suppressed the migration of liver cancer cells (MHCC-97H and HepG2) (Fig. 2D). Moreover, upregulated expression of miR-29c-3p inhibited liver cancer cell (MHCC-97H and HepG2) migration (Fig. 2E).

miR-29c-3p suppresses tumorigenicity in HCC. The role of miR-29c-3p on proliferation in vivo was further studied. In the subcutaneous tumor formation model of nude mice, overexpression of miR-29c-3p inhibited the growth of subcutaneous tumors, which were thinner and lighter in liver cancer cells (MHCC-97H and HepG2) (Fig. 3A-D).

miR-29c-3p directly interacts and inhibits TRIM31. To further understand the specific mechanism of miR-29c-3p in HCC, TargetScan and miRanda were used to predict the target gene of miR-29c-3p, and the results revealed that miR-29c-3p could partially bind to the 3'-UTR of TRIM31 (Fig. 4A). The luciferase reporter assay demonstrated that overexpression of miR-29c-3p significantly inhibited luciferase activity in 
Table III. Univariate and multivariate analysis of various prognostic variables influencing RFS in HCC patients.

\begin{tabular}{|c|c|c|c|c|c|}
\hline \multirow[b]{2}{*}{ Variables } & \multirow[b]{2}{*}{$\mathrm{n}$} & \multicolumn{2}{|c|}{ Univariate analysis } & \multicolumn{2}{|c|}{ Multivariate analysis } \\
\hline & & HR $(95 \% \mathrm{CI})$ & P-value & HR $(95 \%$ CI $)$ & P-value \\
\hline Sex & & $0.672(0.486-1.485)$ & 0.509 & & \\
\hline Male & 34 & & & & \\
\hline Female & 26 & & & & \\
\hline Age (years) & & $0.537(0.389-1.059)$ & 0.543 & & \\
\hline$<50$ & 28 & & & & \\
\hline$\geq 50$ & 32 & & & & \\
\hline Tumor size $(\mathrm{cm})$ & & $0.473(0.571-1.839)$ & 0.005 & $0.634(1.007-2.310)$ & 0.013 \\
\hline$\leq 5$ & 27 & & & & \\
\hline$>5$ & 33 & & & & \\
\hline $\operatorname{AFP}(\mathrm{ng} / \mathrm{ml})$ & & $1.539(1.409-2.847)$ & 0.733 & & \\
\hline$\leq 20$ & 27 & & & & \\
\hline$>20$ & 33 & & & & \\
\hline $\mathrm{HBsAg}$ & & $1.003(0.984-1.530)$ & 0.527 & & \\
\hline Positive & 37 & & & & \\
\hline Negative & 23 & & & & \\
\hline TNM stage & & $1.219(0.347-1.595)$ & 0.010 & $1.180(1.038-2.641)$ & 0.015 \\
\hline $\mathrm{I} / \mathrm{II}$ & 27 & & & & \\
\hline III/IV & 33 & & & & \\
\hline Multiplicity & & $0.495(0.671-1.734)$ & 0.034 & $0.834(0.734-1.995)$ & 0.027 \\
\hline Single & 24 & & & & \\
\hline Multiple $(\geq 2)$ & 36 & & & & \\
\hline Vascular invasion & & $1.045(1.048-3.618)$ & 0.649 & & \\
\hline Presence & 27 & & & & \\
\hline Absence & 33 & & & & \\
\hline Liver cirrhosis & & $1.217(1.257-2.804)$ & 0.806 & & \\
\hline Presence & 32 & & & & \\
\hline Absence & 28 & & & & \\
\hline Intrahepatic metastasis & & $0.673(0.758-2.645)$ & 0.587 & & \\
\hline Presence & 25 & & & & \\
\hline Absence & 35 & & & & \\
\hline miR-29c-3p expression & & $1.148(0.687-1.694)$ & 0.019 & $1.327(1.224-2.647)$ & 0.014 \\
\hline Low & 39 & & & & \\
\hline High & 21 & & & & \\
\hline
\end{tabular}

RFS, recurrence-free survival; HCC, hepatocellular carcinoma.

cells transfected with the wt-3'-UTR of TRIM31, while no significant inhibition was revealed in cells transfected with the mt-3'-UTR of TRIM31 (Fig. 4B). In addition, qRT-PCR and western blot assay results revealed that overexpression of miR-29c-3p inhibited the mRNA and protein expression of TRIM31 in MHCC-97H cells (Fig. 4C and D). The expression of miR-29c-3p was significantly decreased after transfection of the miR-29c-3p inhibitor in MHCC-97H cells (Fig. S1).

miR-29c-3p is highly expressed and negatively correlated with TRIM31 in HCC. The role of TRIM31 in HCC was further explored. qRT-PCR results revealed that TRIM31 was upregulated in HCC tumors compared with paired normal samples (Fig. 5A). Notably, Spearman's correlation analysis revealed that TRIM31 had a negative correlation with miR-29c-3p expression (Fig. 5B). For further experiments, pcDNA-TRIM31 was used to overexpress the expression of TRIM31. qRT-PCR results confirmed that the expression of TRIM31 was significantly increased after transfection of the pcDNA-TRIM31 in liver cancer cells (MHCC-97H and HepG2) (Fig. 5C).

Overexpression of TRIM31 partially abrogates the inhibitory effect of $m i R-29 c-3 p$ in HCC. To verify whether miR-29c-3p 

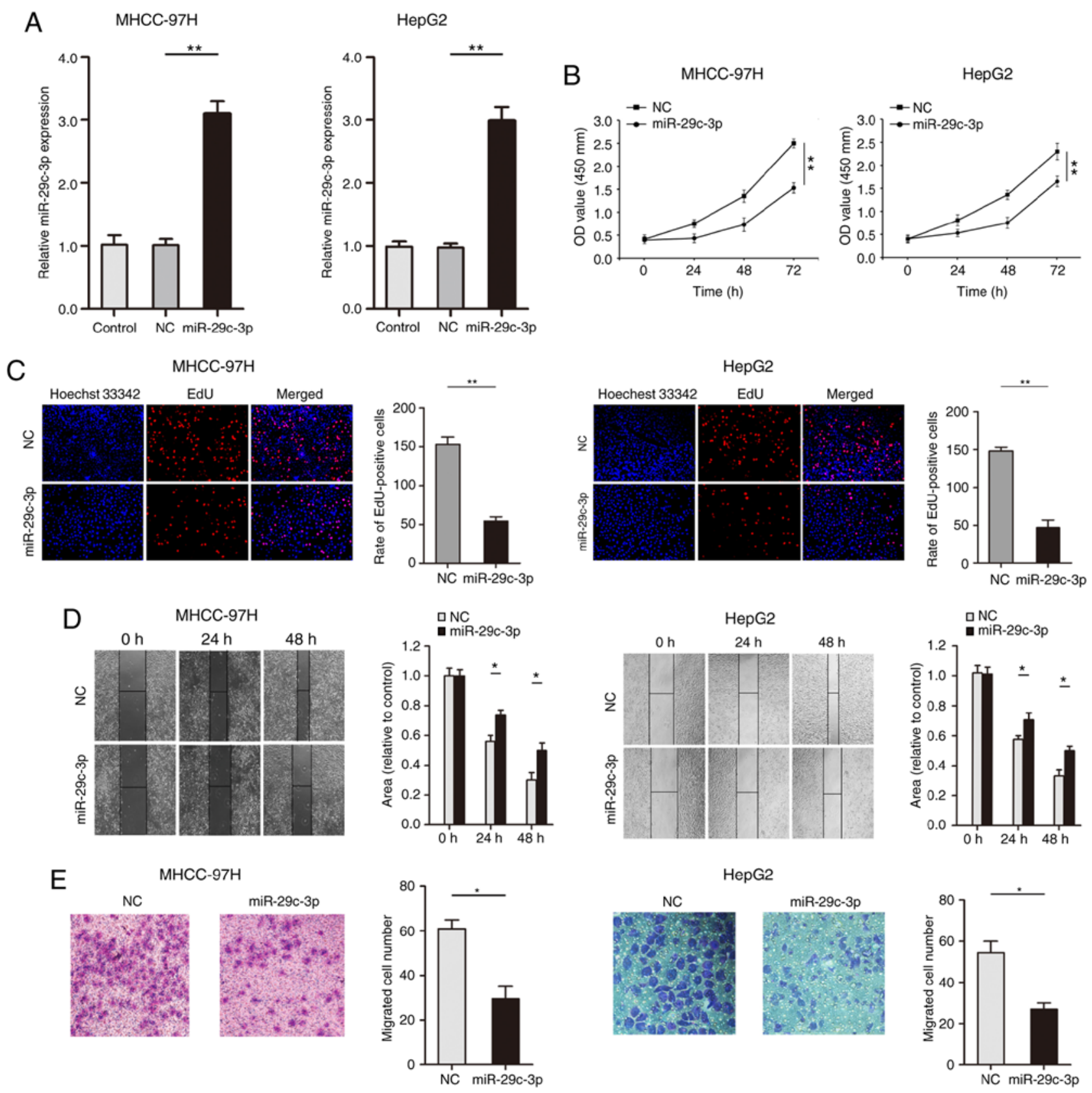

Figure 2. Overexpression of miR-29c-3p inhibits proliferation and migration in HCC cell lines. (A) qRT-PCR detection of miR-29c-3p expression after overexpression of miR-29c-3p in liver cancer cells (MHCC-97H and HepG2). (B) CCK-8 assay of the proliferative ability of HCC cells after overexpression of miR-29c-3p. (C) HCC cell proliferation was detected with EdU after miR-29c-3p overexpression. (D) Wound-healing assays were conducted to determine the effects of overexpression of miR-29c-3p on the migration of HCC cells. (E) Transwell migration assays were used to detect the effects of overexpression of miR-29c-3p on HCC cell migration. ${ }^{*} \mathrm{P}<0.05$ and ${ }^{* *} \mathrm{P}<0.01$ compared to the control. HCC, hepatocellular carcinoma.

plays a biological function through its target gene TRIM31, TRIM31 was overexpressed using pcDNA-TRIM31 in liver cancer cells (MHCC-97H and HepG2) overexpressing miR-29c-3p. Western blot analysis revealed that TRIM31 expression was restored (Fig. 6A). Cellular functional studies reveealed that overexpression of TRIM31 partially abolished the inhibitory effect of miR-29c-3p on the malignant biological behavior of HCC (Fig. 6B-E).

\section{Discussion}

As one of the most significant malignant tumors that threaten human health, HCC causes a large number of deaths every year (2). Therefore, it is urgent to find effective treatments to accurately treat the malignant progression of HCC and improve the quality of life of HCC patients. Numerous studies have confirmed that miRNAs are involved in the malignant progression of HCC and have the ability to predict poor prognosis in HCC patients $(21,22)$. The present study sought to elucidate the potential mechanism of action of miR-29c-3p in HCC. The results revealed that miR-29c-3p was expressed at low levels in $\mathrm{HCC}$ and was closely related to the poor prognosis of patients. Moreover, miR-29c-3p inhibited the proliferation and migration of HCC by directly acting on TRIM31. Collectively, the present findings indicated that miR-29c-3p acts as a tumor suppressor gene by targeting TRIM31 in HCC. 

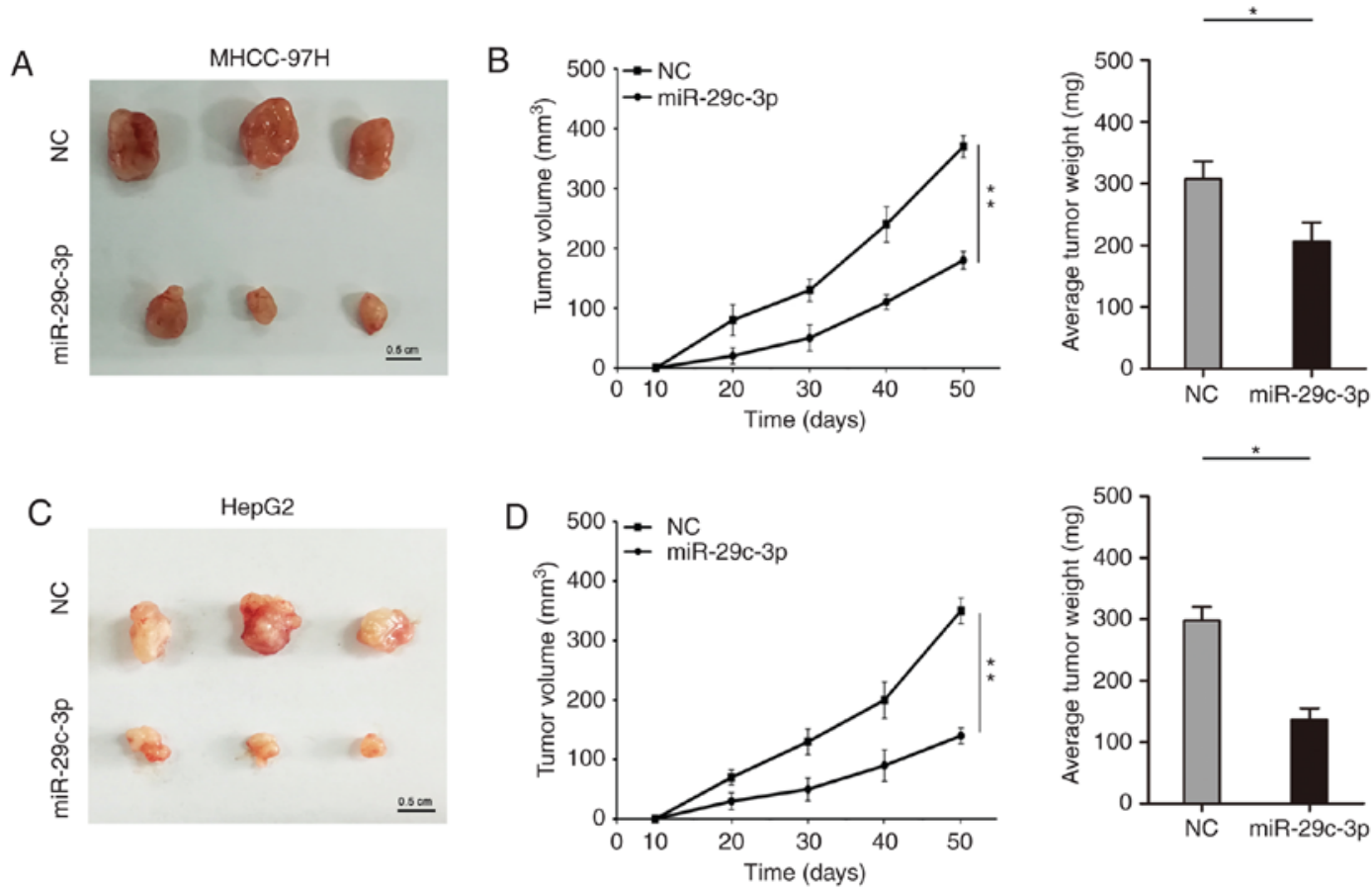

Figure 3. Effect of miR-29c-3p on the tumorigenicity of liver cancer cells (MHCC-97H and HepG2). (A) Observation of the tumor formation of subcutaneously inoculated liver cancer cells (MHCC-97H) overexpressing miR-29c-3p into nude mice (n=3 per group). (B) Analysis of the volume and weight of the subcutaneous tumorigenic masses of liver cancer cells (MHCC-97H). (C) Observation of the tumor formation of subcutaneously inoculated liver cancer cells (HepG2) overexpressing miR-29c-3p into nude mice (n=3 per group). (D) Analysis of the volume and weight of subcutaneous tumorigenic masses of liver cancer cells (HepG2). ${ }^{*} \mathrm{P}<0.05$ and ${ }^{* *} \mathrm{P}<0.01$ compared to the control.
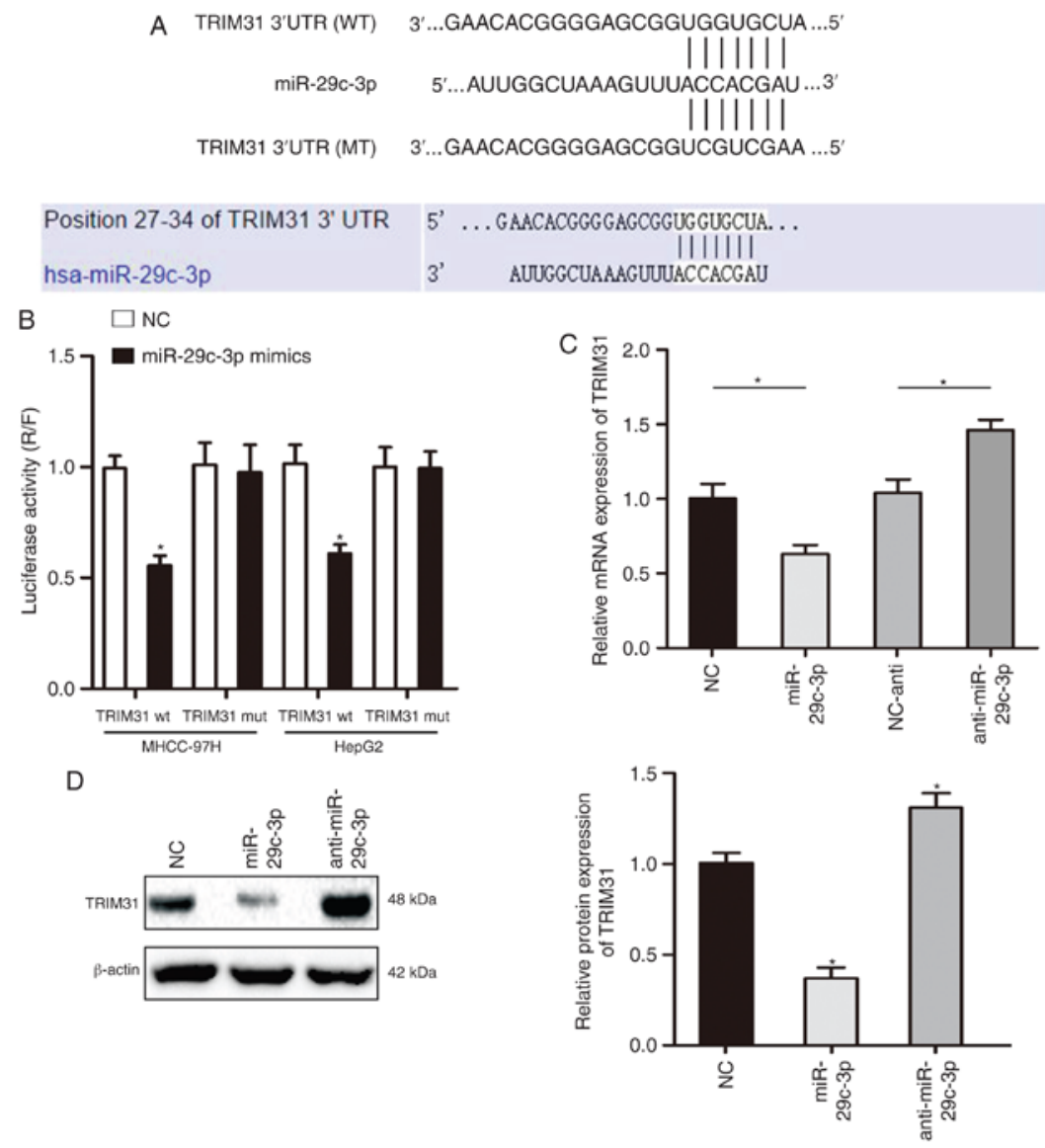

Figure 4. TRIM31 is a direct target gene of miR-29c-3p. (A) TargetScan and miRanda were used to predict the 3'-UTR binding sites of miR-29c-3p and TRIM31. (B) The luciferase activity of wild-type TRIM31-3'UTR (wt) and mutant TRIM31-3'UTR (mut) in HCC cells overexpressing miR-29c-3p in liver cancer cells (MHCC-97H and HepG2). (C and D) qRT-PCR and western blotting were used to analyze the mRNA and protein levels of TRIM31 in MHCC-97H cells that were co-transfected with miR-29c-3p mimics and miR-29c-3p inhibitor. " $\mathrm{P}<0.05$ compared to the control. TRIM31, tripartite motif containing 31 . 
A

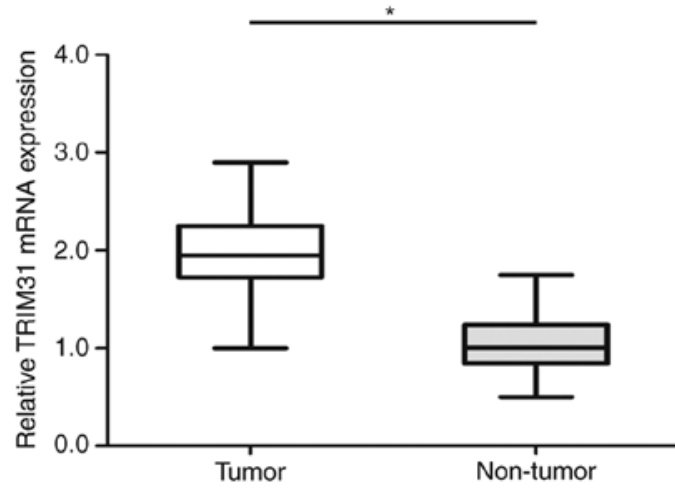

C

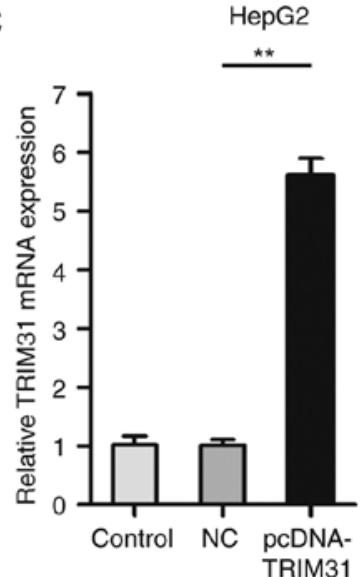

B

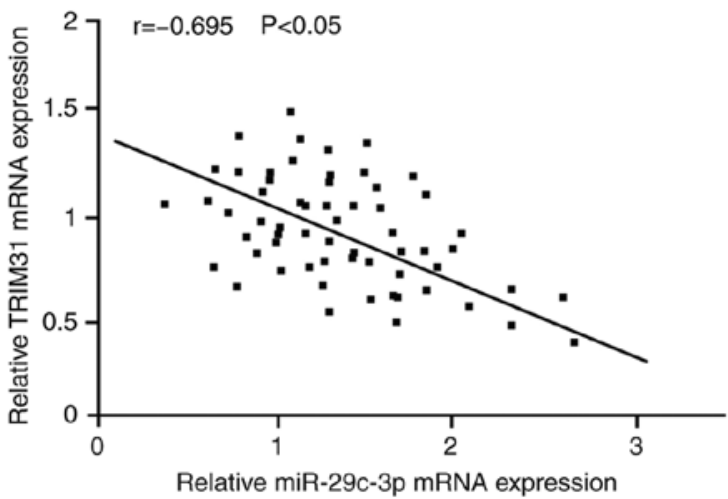

MHCC-97H

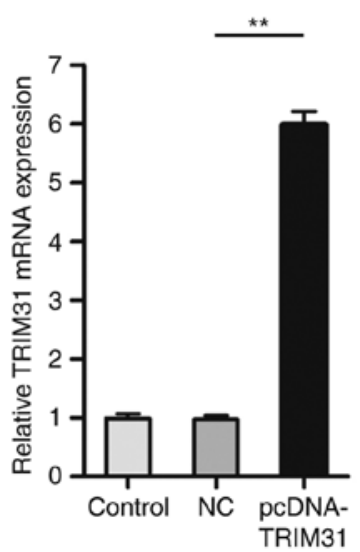

Figure 5. TRIM31 is upregulated in HCC. (A) The results of qRT-PCR revealed the expression level of TRIM31 in HCC and paired normal adjacent tissues. (B) The correlation between miR-29c-3p and TRIM31 expression was evaluated by Spearman's correlation analysis. (C) qRT-PCR detection of TRIM31 expression after overexpression of TRIM31 in liver cancer cells (MHCC-97H and HepG2). ${ }^{*} \mathrm{P}<0.05$ compared to non-tumor tissue; ${ }^{* *} \mathrm{P}<0.01$ compared to the control. HCC, hepatocellular carcinoma; TRIM31, tripartite motif containing 31.

There is evidence that dysfunction of miR-29c-3p promotes malignant development of tumors $(4,23)$. Wu et al revealed that miR-29c-3p regulates the DNA methylation of LATS1 via DNMT3B to influence the Hippo signaling pathway and inhibit the malignant development of HCC (17). Another study also revealed that miR-29c-3p has a binding site in the 3 'UTR of KDM5B and that miR-29c-3p reduces the resistance of endometrial cancer to paclitaxel through KDM5B (24). In gastric cancer, miR-29c-3p regulated malignant development of cancer cells by regulating KIAA1199 expression and activating the FGFR4/Wnt/ $\beta$-catenin and EGFR signaling pathways (15). In the present research results, it was revealed that miR-29c-3p was expressed at low levels in HCC and that patients with decreased expression of miR-29c-3p had a poor prognosis. In addition, overexpression of miR-29c-3p could significantly inhibit the proliferation and migration of HCC.

Previous studies have reported that miR-29c-3p can regulate its expression levels by binding to multiple target genes $(25,26)$. In the present study, the molecular mechanism of miR-29c-3p in the development of HCC was further explored. TRIM31 is a member of the tripartite motif (TRIM) family, and the motif includes three zinc-binding domains, a RING, a B-box type 1 and a B-box type 2, and a coiled-coil region (27). TRIM31 has been revealed to be widely involved in cell proliferation, cell cycle regulation and cell response to viruses and other life processes (28). Through bioinformatics software analysis, it was revealed that TRIM31 is a direct target gene of miR-29c-3p. A recent study reported that TRIM31 improved the resistance of pancreatic cancer to gemcitabine by increasing the K63-linked polyubiquitination of TRAF2 and maintaining the activation of NF- $\kappa B$ to upregulate the level of p65 (29). In addition, high expression of TRIM31 was revealed to activate the PI3K/Akt signaling pathway to promote tumor cell proliferation and invasion in gallbladder cancer (30). In intestinal epithelial cells, TRIM31 interacted directly with phosphatidylethanolamine in a palmitoylation-dependent manner, resulting in the formation of autolysosomes and providing a preventable pathway for the study of intestinal pathogen infections (31). Notably, it was revealed that TRIM31 was highly expressed in HCC, and overexpression of TRIM31 could partially abrogate the inhibitory effect of miR-29c-3p on HCC. In addition, studies have revealed that TRIMs can participate in tumor development in many ways. Most members of the TRIM family have E3 ligase activity. They are directly involved in the specific recognition of target molecules by acting on the skeleton protein between E3 and the enzyme (32). TRIMs play an important role in numerous tumor-related signaling pathways, such as the NF-kB signaling pathway and MAPK signaling pathway $(33,34)$. Based on the aforementioned results and previous studies, we will further explore the 
A
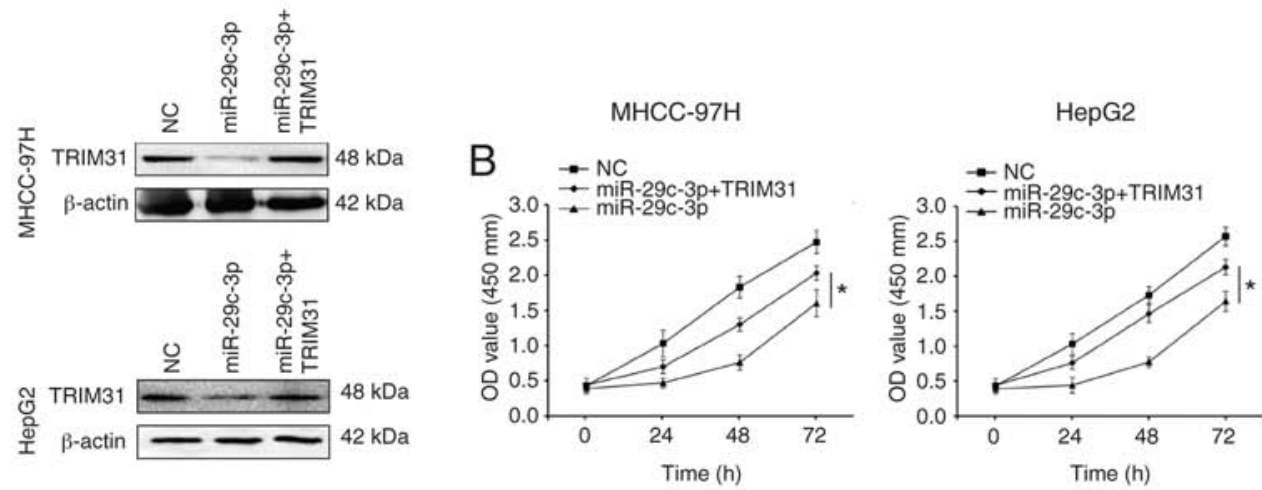

C
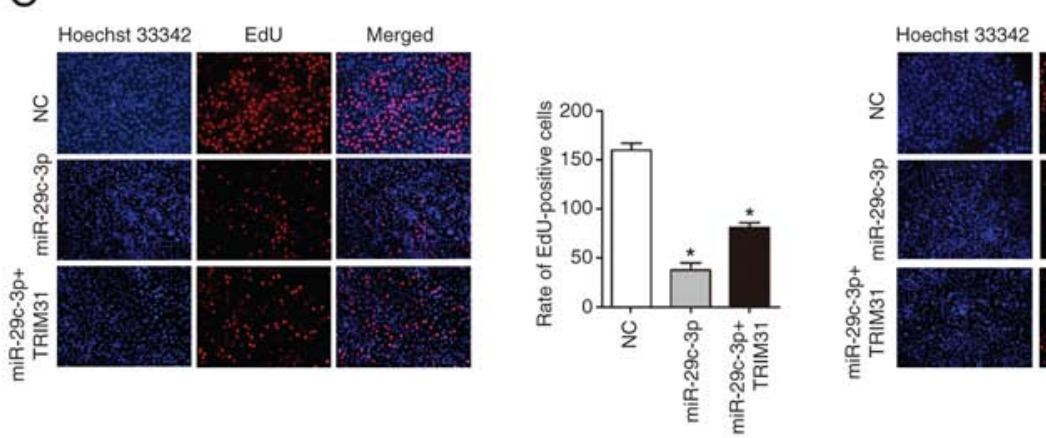

HepG2

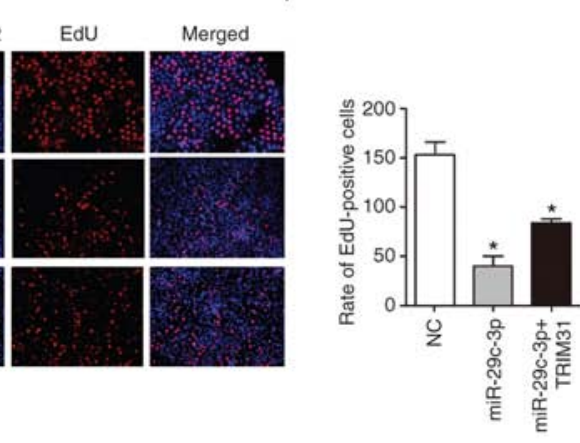

D

MHCC-97H

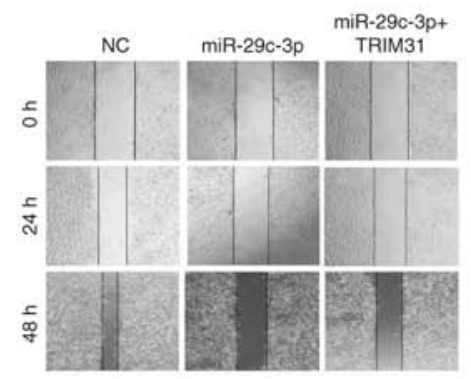

$E$

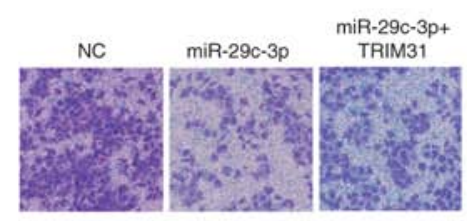

MHCC-97H
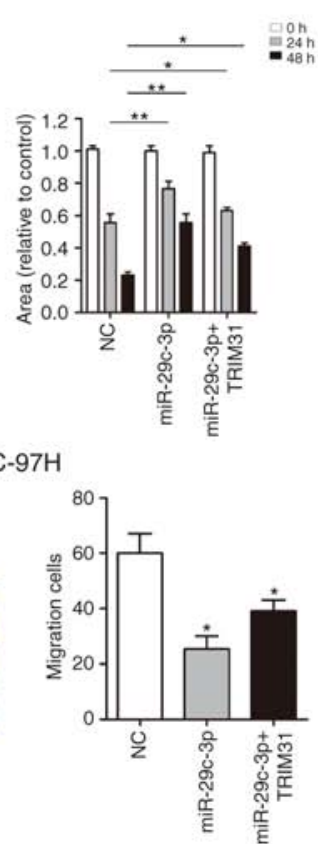

HepG2

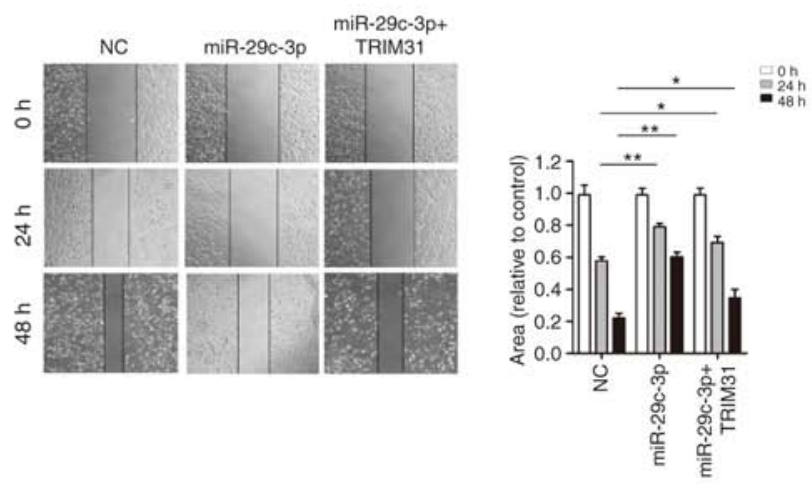

HepG2

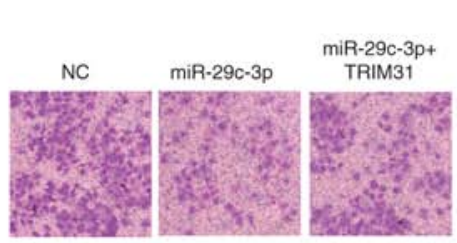

Figure 6. Overexpression of TRIM31 partially attenuates the inhibitory effect of miR-29c-3p on proliferation and migration in HCC. (A) Western blot detection of TRIM31 expression after overexpression of TRIM31 in liver cancer cells (MHCC-97H-miR-29c-3p and HepG2-miR-29c-3p). (B) CCK-8 assay analysis of the proliferative ability of HCC cells after overexpression of TRIM31 in liver cancer cells (MHCC-97H-miR-29c-3p and HepG2-miR-29c-3p). (C) HCC cell proliferation was detected by EdU after overexpression of TRIM31 in liver cancer cells (MHCC-97H-miR-29c-3p and HepG2-miR-29c-3p). (D) Wound-healing assays were conducted to determine the effects of overexpression of TRIM31 on the migration of HCC cells. (E) Transwell migration assays were used to detect the effects of TRIM31 overexpression on HCC cell migration. ${ }^{*} \mathrm{P}<0.05$ and ${ }^{* *} \mathrm{P}<0.01$ compared to the control. HCC, hepatocellular carcinoma; TRIM31, tripartite motif containing 31.

specific molecular mechanism of miR-29c-3p and TRIM31in HCC.

In conclusion, the present results demonstrated that miR-29c-3p could inhibit the proliferation and migration of
HCC by targeting TRIM31, and may be used as an important prognostic indicator for HCC patients. Further study of the specific molecular mechanism of the miR-29c-3p/TRIM31 axis will provide new hope for the diagnosis and treatment of HCC. 


\section{Acknowledgements}

We thank Dr Zhenru Wu and Dr Yongjie Zhou for pathology technique assistance and animal model establishing.

\section{Funding}

The present study was supported by grants from the National Natural Science Foundation of China (NM. 81470037 and 817700653), the '1.3.5 Project for Disciplines of Excellence, West China Hospital, Sichuan University (ZY2017308), and the Projects of the Ministry of Science and Technology (2017ZX10203205-005-002).

\section{Availability of data and materials}

The datasets used during the present study are available from the corresponding author upon reasonable request.

\section{Authors' contributions}

TL and JY acquired the data and created a draft of the manuscript. TL and LJ prepared the experimental materials and performed the in vitro assays. TL, LJ and LK interpreted the data, performed the statistical analysis and analyzed the results. TL and JY revised and approved the final version of the manuscript. All authors read and approved the manuscript and agree to be accountable for all aspects of the research in ensuring that the accuracy or integrity of any part of the work are appropriately investigated and resolved.

\section{Ethics approval and consent to participate}

The protocol of the present study was approved by the Institutional Research Ethics Committee of West China Hospital of Sichuan University and informed consent was obtained from every patient enrolled in this study. The protocols regarding the in vivo manipulations were approved by the Animal Care Ethics Committee of West China Hospital of Sichuan University.

\section{Patient consent for publication}

Not applicable.

\section{Competing interests}

The authors declare that they have no competing interests.

\section{References}

1. Siegel RL, Miller KD and Jemal A: Cancer statistics, 2017. CA Cancer J Clin 67: 7-30, 2017.

2. Greten TF, Wang XW and Korangy F: Current concepts of immune based treatments for patients with HCC: From basic science to novel treatment approaches. Gut 64: 842-848, 2015.

3. Chen W, Zheng R, Baade PD, Zhang S, Zeng H, Bray F, Jemal A, Yu XQ and He J: Cancer statistics in China, 2015. CA Cancer J Clin 66: 115-132, 2016.

4. Li Z and Rana TM: Therapeutic targeting of microRNAs: Current status and future challenges. Nat Rev Drug Discov 13: 622-638, 2014

5. Dragomir MP, Knutsen E and Calin GA: SnapShot: Unconventional miRNA functions. Cell 174: 1038-1038.e1, 2018.
6. Calin GA and Croce CM: MicroRNA-cancer connection: The beginning of a new tale. Cancer Res 66: 7390-7394, 2006.

7. Hayes $\mathrm{CN}$ and Chayama K: MicroRNAs as biomarkers for liver disease and hepatocellular carcinoma. Int J Mol Sci 17: 280, 2016.

8. Du H, Xu Q, Xiao S, Wu Z, Gong J, Liu C, Ren G and Wu H: MicroRNA-424-5p acts as a potential biomarker and inhibits proliferation and invasion in hepatocellular carcinoma by targeting TRIM29. Life Sci 224: 1-11, 2019.

9. Tang H, Lv W, Sun W, Bi Q and Hao Y: miR-505 inhibits cell growth and EMT by targeting MAP3K3 through the AKT-NFKB pathway in NSCLC cells. Int J Mol Med 43: 1203-1216, 2019.

10. Yang L, Zhang S, Guo K, Huang H, Qi S, Yao J and Zhang Z: miR-125a restrains cell migration and invasion by targeting STAT3 in gastric cancer cells. Onco Targets Ther 12: 205-215, 2018.

11. Dong XZ, Song Y, Lu YP, Hu Y, Liu P and Zhang L: Sanguinarine inhibits the proliferation of BGC-823 gastric cancer cells via regulating $\mathrm{miR}-96-5 \mathrm{p} / \mathrm{miR}-29 \mathrm{c}-3 \mathrm{p}$ and the MAPK/JNK signaling pathway. J Nat Med 73: 777-788, 2019.

12. Chen G, Zhou T, Li Y, Yu Z and Sun L: p53 target miR-29c-3p suppresses colon cancer cell invasion and migration through inhibition of PHLDB2. Biochem Biophys Res Commun 487: 90-95, 2017.

13. Lu Y, Tang L, Zhang Z, Li S, Liang S, Ji L, Yang B, Liu Y and Wei W: Long noncoding RNA TUG1/miR-29c axis affects cell proliferation, invasion, and migration in human pancreatic cancer. Dis Markers 22: 6857042, 2018.

14. Fang R, Huang Y, Xie J, Zhang J and Ji X: Downregulation of miR-29c-3p is associated with a poor prognosis in patients with laryngeal squamous cell carcinoma. Diagn Pathol 14: 109, 2019.

15. Wang L, Yu T, Li W, Li M, Zuo Q, Zou Q and Xiao B: The miR-29c-KIAA1199 axis regulates gastric cancer migration by binding with WBP11 and PTP4A3. Oncogene 38: 3134-3150, 2019.

16. Zhang S, Jin J, Tian X and Wu L: sa-miR-29c-3p regulates biological function of colorectal cancer by targeting SPARC. Oncotarget 8: 104508-104524, 2017.

17. Wu H, Zhang W, Wu Z, Liu Y, Shi Y, Gong J, Shen W and Liu C: miR-29c-3p regulates DNMT3B and LATS1 methylation to inhibit tumor progression in hepatocellular carcinoma. Cell Death Dis 18: 48, 2019.

18. Lopes CB, Magalhães LL, Teófilo CR, Alves APNN, Montenegro RC,Negrini M and Ribeiro-Dos-Santos Â:Differential expression of hsa-miR-221, hsa-miR-21, hsa-miR-135b, and hsa-miR-29c suggests a field effect in oral cancer. BMC Cancer 18: $721,2018$.

19. Li W, Yi J, Zheng X, Liu S, Fu W, Ren L, Li L, Hoon DSB, Wang J and Du G: miR-29c plays a suppressive role in breast cancer by targeting the TIMP3/STAT1/FOXO1 pathway. Clin Epigenetics 10: 64, 2018.

20. Livak KJ and Schmittgen TD: Analysis of relative gene expression data using real-time quantitative PCR and the 2(-Delta Delta C(T)) method. Methods 25: 402-408, 2001.

21. Chhabra R: miRNA and methylation: A multifaceted liaison. Chembiochem 16: 195-203, 2015.

22. Rupaimoole R and Slack FJ: MicroRNA therapeutics: Towards a new era for the management of cancer and other diseases. Nat Rev Drug Discov 16: 203-222, 2017.

23. Wang $\mathrm{H}$, Zhu Y, Zhao M, Wu C, Zhang P, Tang L, Zhang H, Chen X, Yang Y and Liu G: miRNA-29c suppresses lung cancer cell adhesion to extracellular matrix and metastasis by targeting integrin beta1 and matrix metalloproteinase2 (MMP2). PLoS One 8: e70192, 2013.

24. Li L, Shou H, Wang Q and Liu S: Investigation of the potential theranostic role of $\mathrm{KDM} 5 \mathrm{~B} / \mathrm{miR}-29 \mathrm{c}$ signaling axis in paclitaxel resistant endometrial carcinoma. Gene 694: 76-82, 2019.

25. Morita S, Horii T, Kimura M, Ochiya T, Tajima S and Hatada I: miR-29 represses the activities of DNA methyltransferases and DNA demethylases. Int J Mol Sci 14: 14647-14658, 2013.

26. Liu L, Bi N, Wu L, Ding X, Men Y, Zhou W, Li L, Zhang W, Shi S, Song Y and Wang L: MicroRNA-29c functions as a tumor suppressor by targeting VEGFA in lung adenocarcinoma. Mol Cancer 16: 50, 2017.

27. Reymond A, Meroni G, Fantozzi A, Merla G, Cairo S, Luzi L, Riganelli D, Zanaria E, Messali S, Cainarca S, et al: The tripartite motif family identifies cell compartments. EMBO J 20: 2140-2151, 2001.

28. Song H, Liu B, Huai W, Yu Z, Wang W, Zhao J, Han L, Jiang G, Zhang L, Gao C and Zhao W: The E3 ubiquitin ligase TRIM31 attenuates NLRP3 inflammasome activation by promoting proteasomal degradation of NLRP3. Nat Commun 7: 13727, 2016. 
29. Yu C, Chen S, Guo Y and Sun C: Oncogenic TRIM31 confers gemcitabine resistance in pancreatic cancer via activating the NF- $\kappa$ B signaling pathway. Theranostics 8: 3224-3236, 2018.

30. Li H,Zhang Y,Hai J, Wang J,Zhao B, Du L and Geng X: Knockdown of TRIM31 suppresses proliferation and invasion of gallbladder cancer cells by down-regulating MMP2/9 through the PI3K/Akt signaling pathway. Biomed Pharmacother 103: 1272-1278, 2018.

31. Ra EA, Lee TA, Won Kim S, Park A, Choi HJ, Jang I, Kang S, Hee Cheon J, Cho JW, Eun Lee J, et al: TRIM31 promotes Atg5/Atg7-independent autophagy in intestinal cells. Nat Commun 7: 11726, 2016.
32. Scott DC, Sviderskiy VO, Monda JK, Lydeard JR, Cho SE, Harper JW and Schulman BA: Structure of a RING E3 trapped in action reveals ligation mechanism for the ubiquitin-like protein NEDD8. Cell 157: 1671-1684, 2014.

33. Hatakeyama S: TRIM proteins and cancer. Nat Rev Cancer 11: 792-804, 2011.

34. Sato T, Takahashi H, Hatakeyama S, Iguchi A and Ariga T: The TRIM-FLMN protein TRIM45 directly interacts with RACK and negatively regulates PKC-mediated signaling pathway. Oncogene 34: 1280-1291, 2015.

(i) (9) This work is licensed under a Creative Commons Attribution-NonCommercial-NoDerivatives 4.0 International (CC BY-NC-ND 4.0) License. 